DOI: $10.17516 / 1997-1397-2020-13-5-574-582$

УДК 517.9

\title{
Layered Motion of Two Immiscible Liquids with a Free Boundary
}

\author{
Elena N. Lemeshkova* \\ Institute of computational modelling SB RAS \\ Krasnoyarsk, Russian Federation
}

Received 26.05.2020, received in revised form 06.06.2020, accepted 17.07.2020

\begin{abstract}
The unidirectional motion of two viscous immiscible incompressible liquids in a flat channel is studied. An unsteady temperature gradient is set on the bottom solid wall, and the upper wall is a free boundary. Liquids contact on a flat interface. The motion is caused by the combined action of thermogravitational and thermocapillary forces and a given total unsteady flow rate in the layers. The corresponding initial boundary value problem is conjugate and inverse, since the pressure gradient along the channel is determined together with the velocity and temperature field. An exact stationary solution was found for it. In Laplace images, the solution of the non-stationary problem is found in the quadrature forms. It was established that if the temperature on the bottom wall and the flow rate stabilize with time, then the motion goes to a stationary state with time. This fact indicates the stability of the stationary solution with respect to unidirectional unsteady perturbations. The calculation results showing various methods of controlling motion by setting the temperature on the wall are given.
\end{abstract}

Keywords: thermocapillary, interface, Oberbek-Boussinesq equations.

Citation: E.N. Lemeshkova, Layered Motion of Two Immiscible Liquids with a Free Boundary, J. Sib. Fed. Univ. Math. Phys., 2020, 13(5), 574-582. DOI: 10.17516/1997-1397-2020-13-5-574-582.

It is well known that in a nonuniformly heated pure liquid medium there occurs a motion which is called convection. Under the condition close to zero gravity, the inhomogeneity of the temperature field affects the flow from the region of little to the region of large surface tension due to a spontaneous decrease of the interface free energy between two liquid media or the free boundary (Marangoni effect, see the detailed review in [1]). Observation of thermocapillary motion due to interfacial tension gradients in terrestrial conditions is very difficult, since gravitational convection becomes the dominant form of motion. However, in recent years in connection with the development of modern technologies, new problems have arisen in which it is necessary to take into account the thermocapillary effect in terrestrial conditions. For example, in laser annealing of semiconductors or in laser processing of materials with fusion, which is used to alloy the surface layer of metal [2]. In this case, relatively long thin layers of melt (of the order of several micrometers) appear on the surface of materials, in which, according to [3,4], thermocapillary forces dominate over gravitational forces. Knowledge of the laws of thermal convection in liquid layered systems is of interest for understanding the hydrodynamics and heat and mass transfer processes when applying multilayer coatings, in thermal stabilization systems of power plants or cooling electronic devices, in the processes of growing single crystals and films, etc. [5]. It is known that the solution of R.V. Birikh [6] describes a stationary convective flow in a strip. For the first time, its generalization to the case when the longitudinal temperature

*elena_cher@icm.krasn.ru https://orcid.org/0000-0002-9059-2876

(c) Siberian Federal University. All rights reserved 
gradient depends on time was proposed by V.V.Pukhnachev in [7], as well as [8]. A similar generalization the solution of the problem of the motion of two immiscible liquids with a flat interface allows [9]. In the monograph [10], the problems of unidirectional motion of a viscous heat-conducting fluid in a flat horizontal channel in a field of gravity acting across the channel are studied. At the same time, an unsteady temperature field is set on a fixed solid wall, and the upper boundary can be a solid wall, either insulated or not, or a free boundary. The present work is devoted to the study of the unidirectional thermogravitational motion of two immiscible viscous liquids in a flat horizontal channel. In this case, an unsteady temperature field, which is linear in the longitudinal coordinate, is set on the lower solid wall and the upper wall is a free boundary. The system of Oberbek-Boussinesq equations is taken as a mathematical model. A detailed conclusion and analysis of the main assumptions that lead to this system are available in many works, for example, in [7].

\section{Statement of problem}

We consider a system of two incompressible immiscible liquids with an interface $y=0$. The parameters of the fluid that moves in the strip $-h_{1}<y<0,-\infty<x<\infty$ are denoted by index "1", and the parameters of the fluid moving in the strip $0<y<h_{2}$ will be denoted by index "2"; $\rho_{j}, \nu_{j}, \mu_{j}, \chi_{j}, \beta_{j}$ are densities, kinematic viscosities, dynamic viscosities, thermal diffusivity and volume expansion coefficients, respectively. Further on, it is assumed that these parameters are positive constants. Let $x$ and $y$ denote the horizontal and vertical coordinates, gravity with acceleration $g$ acts in the negative direction of the $y$ axis. Substitution of a solution of the form [8]

$$
u_{j}=w_{j}(y, t), \quad \theta_{j}=-a_{j}(y, t) x+T_{j}(y, t), \quad p_{j}=-b_{j}(y, t) x+P_{j}(y, t), \quad j=1,2 .
$$

into the Oberbek-Boussinesq system leads to the equations

$$
\begin{aligned}
& a_{j t}=\chi_{j} a_{j y y}, \\
& b_{j y}=\rho_{j} g \beta_{j} a_{j}, \\
& w_{j t}=\nu_{j} w_{j y y}+\frac{1}{\rho_{j}} b_{j}, \\
& T_{j t}=\chi_{j} T_{j y y}+a_{j} w_{j}, \\
& P_{j y}=\rho_{j} g \beta_{j} T_{j} .
\end{aligned}
$$

In (1) $u_{j}$ is the projection of the velocity vector on the $x$ axis, $\theta_{j}$ is temperature, $p_{j}$ is pressure deviation from hydrostatic one. Next, we consider the problem only for determining the velocity field, that is, the problem for $w_{j}$ and $a_{j}$. The functions $T_{j}$ will be the solution of the conjugate problem similar to the problem for $a_{j}$. Using the known functions $a_{j}$ and $T_{j}$, the functions $b_{j}(y, t)$ and $P_{j}(y, t)$ are reconstructed by quadratures from the second and fifth equations of (2)

$$
\begin{gathered}
b_{j}(y, t)=\rho_{j} g \beta_{j} \int_{0}^{y} a_{j}(z, t) d z+C_{j}(t), \\
P_{j}(y, t)=\rho_{j} g \beta_{j} \int_{0}^{y} T_{j}(z, t) d z+P_{0 j}(t) .
\end{gathered}
$$

For the system (2), we can pose various initial-boundary value problems that describe the motion in a flat channel: one liquid in a channel with solid impermeable walls, a solid wall and a free 
boundary, one or more interfaces, an interface and a free boundary (a part of the statements of such problems was considered in $[8,10])$. In this paper, we consider the last case for a specific problem, namely, when the temperature is set on the solid lower wall $y=-h_{1}$, and the upper wall $y=h_{2}$ is free boundary. Since the interface $y=0$ at the initial moment of time is assumed to be horizontal, at any moment of time it will be such [10, p. 41]. The large capillary pressure (Weber number We $\gg 1$ ) allows us to assume that the free surface $y=h_{2}$ also remains flat [11].

We introduce the characteristic length scales for the first and second layer, these are $h_{1}$ and $h_{2}$, for time this is $h_{1}^{2} \chi_{1}^{-1}$ and functions $w_{j}, a_{j}, C_{j}$ these are $\chi_{j} h_{j}^{-1}, \tilde{a}=\max _{t \geqslant 0}\left|a_{1}\left(-h_{1}, t\right)\right|>0$, $\mu_{1} \chi_{1} h_{1}^{-3}$ and write out the conjugate initial boundary value problem for the functions $a_{j}(y, t)$ in the dimensionless form

$$
\begin{gathered}
a_{1 t}(y, t)=a_{1 y y}(y, t), \quad-1<y<0, \\
a_{2 t}(y, t)=h^{2} \chi^{-1} a_{2 y y}(y, t), \quad 0<y<1, \\
a_{j}(y, 0)=a_{j 0}(y), \\
a_{1}(-1, t)=a(t), \quad a_{2 y}(1, t)+\operatorname{Bi}_{2}(1, t)=0, \\
a_{1}(0, t)=a_{2}(0, t), \quad k a_{1 y}(0, t)=h a_{2 y}(0, t) .
\end{gathered}
$$

In (4)-(7) $h=h_{1} / h_{2}, \chi=\chi_{1} / \chi_{2}, k=k_{1} / k_{2}, \mathrm{Bi}=\gamma h_{2} k_{2}^{-1}$ is the Bio number, $\gamma \geqslant 0$ is the interfacial heat transfer coefficient, and functions $a_{j 0}(y)$ and $a(t)$ are known. Relations (7) follow from the equality of temperatures and heat fluxes at the interface $y=0$.

Let us pass to the formulation of the problem for dimensionless velocities $w_{j}(y, t)$

$$
\begin{gathered}
\mathrm{P}_{1}^{-1} w_{1 t}(y, t)=w_{1 y y}(y, t)+\operatorname{Ra}_{1} \int_{0}^{y} a_{1}(z, t) d z+C_{1}(t), \quad-1<y<0, \\
\chi h^{-2} \mathrm{P}_{2}^{-1} w_{2 t}(y, t)=w_{2 y y}(y, t)+\operatorname{Ra}_{2} \int_{0}^{y} a_{2}(z, t) d z+\mu \chi h^{-3} C_{2}(t), \quad 0<y<1, \\
w_{j}(y, 0)=w_{j 0}(y), \\
w_{1}(-1, t)=0, \quad \chi h^{-1} w_{1}(0, t)=w_{2}(0, t) .
\end{gathered}
$$

The first condition in (11) is the sticking condition on the lower solid fixed wall $y=-1$, and the second is a consequence of the continuity of velocities at the interface $y=0$. The dynamic condition on the interface $y=0$ is reduced to two:

$$
w_{2 y}(0, t)-\mu \chi h^{-2} w_{1 y}(0, t)=-\mathrm{Ma}_{1} a_{1}(0, t), \quad p_{1}(0, t)=p_{2}(0, t) .
$$

The last condition, together with representations (1), (3), implies the equalities

$$
C_{1}(t)=C_{2}(t) \equiv C(t), \quad P_{1}(0, t)=P_{2}(0, t) .
$$

Besides, on the free boundary $y=1$, from the dynamic condition for tangential stresses it follows

$$
w_{2 y}(1, t)=\mathrm{Ma}_{2} a_{2}(1, t) .
$$

Assuming We $\gg 1$, from the condition for normal stresses we obtain that the free surface remains flat. In equations (8), (9) and in conditions (12), (13), dimensionless parameters arise $\mu=\mu_{1} / \mu_{2}$, 
$\mathrm{P}_{j}=\nu_{j} / \chi_{j}$ are the Prandtl numbers, $\mathrm{Ra}_{j}=g \beta_{j} \tilde{a} h_{j}^{4} / \nu_{j} \chi_{j}$ are the Rayleigh numbers, $\mathrm{Ma}_{j}=$ $=æ_{j} \tilde{a} h_{2}^{2} / \mu_{2} \chi_{2}$ are the Marangoni numbers.

If $C(t)$ is given, then the statement of the problem for velocities is completed. The aim of this work is to study the inverse problem, therefore, it is necessary to put another condition is the total consumption in layers

$$
\chi \int_{-1}^{0} w_{1}(z, t) d z+\int_{0}^{1} w_{2}(z, t) d z=q(t) .
$$

\section{Stationary solution}

Suppose that $a(t)=a_{0}=$ const, $q(t)=q_{0}=$ const. We give the form of a stationary solution $a_{j}^{0}(y), w_{j}^{0}(y)$ and $C^{0}=$ const

$$
\begin{gathered}
a_{1}^{0}(y)=-\frac{\operatorname{Bi} a_{0}}{\delta}(y+1)+a_{0}, \quad-1 \leqslant y \leqslant 0, \\
a_{2}^{0}(y)=-\frac{\operatorname{Bi} a_{0}}{\delta}\left(\frac{k}{h} y+1\right)+a_{0}, \quad 0 \leqslant y \leqslant 1, \\
w_{1}^{0}(y)=-\frac{a_{0} \operatorname{Ra}_{1}}{24}\left(-\frac{\mathrm{Bi}}{\delta} y^{4}+4\left(1-\frac{\mathrm{Bi}}{\delta}\right) y^{3}\right)-\frac{C^{s}}{2} y^{2}+m_{1} y+m_{2}, \quad-1 \leqslant y \leqslant 0, \\
w_{2}^{0}(y)=-\frac{a_{0} \operatorname{Ra}_{2}}{24}\left(-\frac{k \mathrm{Bi}}{h \delta} y^{4}+4\left(1-\frac{\mathrm{Bi}}{\delta}\right) y^{3}\right)-\frac{\mu \chi C^{s}}{2 h^{3}} y^{2}+m_{3} y+m_{4}, \quad 0 \leqslant y \leqslant 1, \\
C^{s}=\delta_{1}+h m_{1} .
\end{gathered}
$$

In (15), (16) we introduced the notation

$$
\begin{gathered}
\delta=\frac{(1+\mathrm{Bi}) k}{h}+\mathrm{Bi} \\
\delta_{1}=-\frac{a_{0} h^{3}}{\mu \chi}\left[\frac{\mathrm{Ra}}{6}\left(3-\frac{\mathrm{Bi}}{\delta}\left(3+\frac{k}{h}\right)\right)+\mathrm{Ma}_{1}\left(1-\frac{\mathrm{Bi}}{\delta}\right)+\mathrm{Ma}_{2}\left(1-\frac{\mathrm{Bi}}{\delta}\left(1+\frac{k}{h}\right)\right)\right] \\
\delta_{2}=\frac{a_{0} \mathrm{Ra}_{1}}{24}\left(\frac{3 \mathrm{Bi}}{\delta}-4\right)+\frac{\delta_{1}}{2}, \quad \delta_{3}=\frac{\chi a_{0} \mathrm{Ra}_{1}}{120}\left(5-\frac{4 \mathrm{Bi}}{\delta}\right)-\frac{a_{0} \mathrm{Ra}_{2}}{120}\left(5-\frac{\mathrm{Bi}}{\delta}\left(5+\frac{k}{h}\right)\right), \\
m_{1}=\frac{-6 \delta_{3}+\chi \delta_{1}\left(1+\mu h^{-3}\right)+3 a_{0} \mathrm{Ma}_{1}\left(1-\mathrm{Bi} \delta^{-1}\right)-6 \delta_{2} \chi\left(1+h^{-1}\right)+6 q_{0}}{-h \chi\left(1+\mu h^{-3}\right)-3 \chi\left(1-\mu h^{-2}\right)+3 h^{-1} \chi(h+1)(h+2)} \\
m_{2}=\delta_{2}+\frac{m_{1}}{2}(h+2), \quad m_{3}=\frac{\mu \chi}{h^{2}} m_{1}-a_{0} \mathrm{Ma}_{1}\left(1-\frac{\mathrm{Bi}}{\delta}\right), \quad m_{4}=\frac{\chi}{h} m_{2},
\end{gathered}
$$

Fig. 1 shows stationary velocity profiles $w_{j}^{0}(y)$ depending on the dimensionless flow rate $q_{0}$. Hereinafter, the function $w^{0}(y)$ coincides with the functions $w_{j}^{0}(y), j=1,2$ on their domains of definition. The calculations show that with an increase in $q_{0}$, the velocity profile in the first layer becomes linear, i.e., a Couette flow arises. In the second layer, a Poiseuille flow arises (parabolic profile). With a decrease in the layers $q_{0}$, zones of the return flow arise.

Fig. 2 shows stationary velocity profiles for different values of the Bio number. It can be seen that in the second layer for $\mathrm{Bi}=0$ (a thermally insulated free surface) the velocity profile is linear, and with an increase the Bio number it becomes parabolic. The dependence of the velocity profile in the layers on the dimensionless temperature gradient $a_{0}$ is shown in Fig. 3 . 


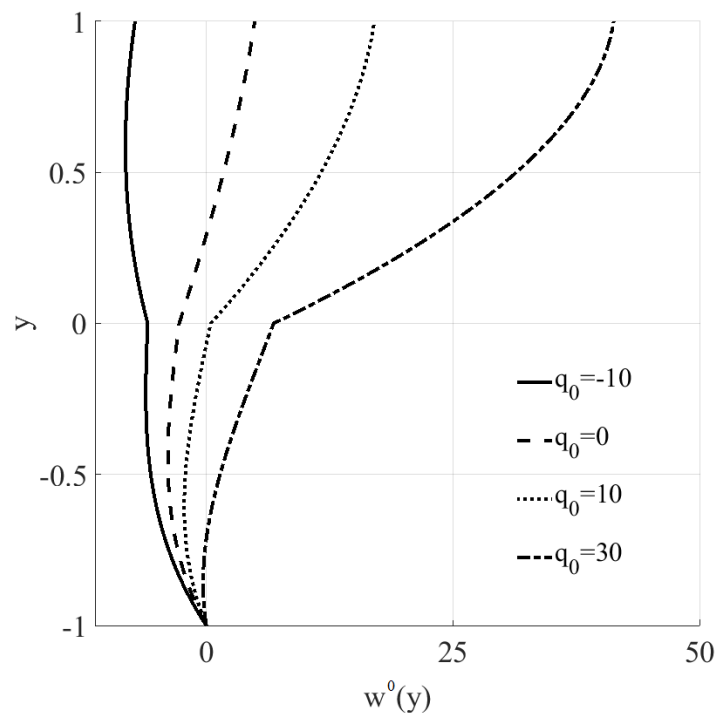

Fig. 1. Stationary profile of velocities $w_{j}^{0}(y)$ depending on the dimensionless flow rate

The case when $a_{0}<0$ means that the bottom wall is cooling. So, for $a_{0}=2$ a return flow arises near the interface $y=0$, and for $a_{0}=-2$ the direction of the current changes to the opposite. At $a_{0}=0$ in both layers the velocities have a parabolic profile and there are no zones of return flow.

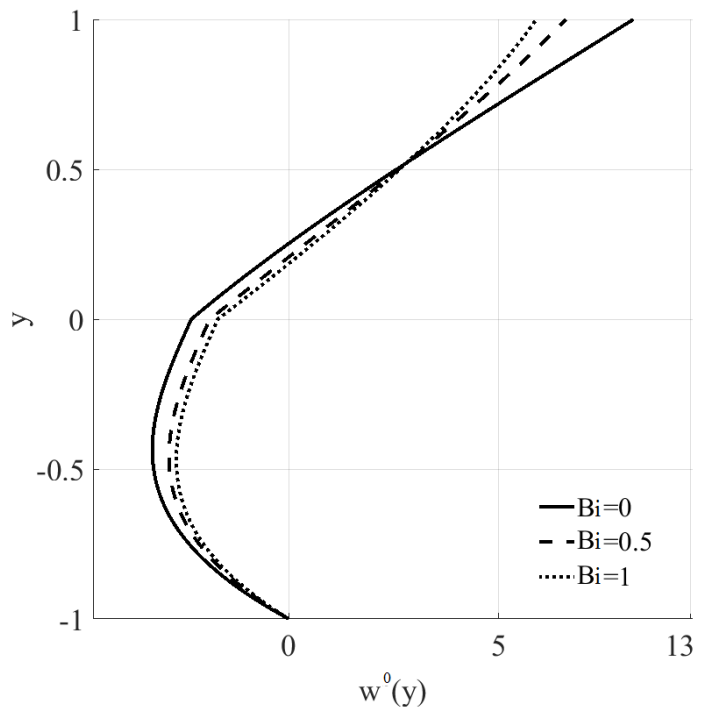

Fig. 2. Stationary profile of velocities $w_{j}^{0}(y)$ depending on the Bio number

Changes of the Marangoni numbers also affect the nature and intensity of the arising currents. So, a change in the number $\mathrm{Ma}_{1}$ affects the direction and intensity of the flow near the interface $y=0$ (see Fig. 4 a), and the number $\mathrm{Ma}_{2}$ only affects the intensity near the free boundary (see Fig. 4 b). 


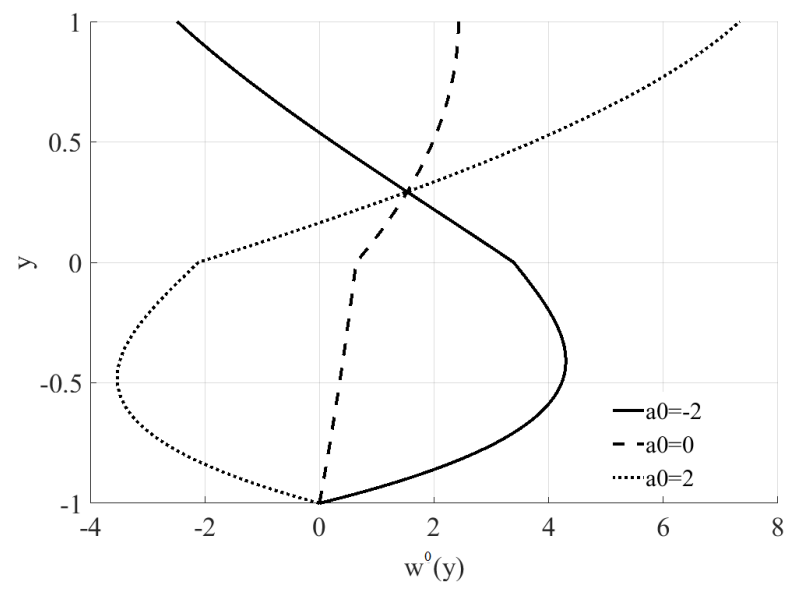

Fig. 3. Stationary profile of velocities $w_{j}^{0}(y)$ depending on the dimensionless temperature gradient $a_{0}$

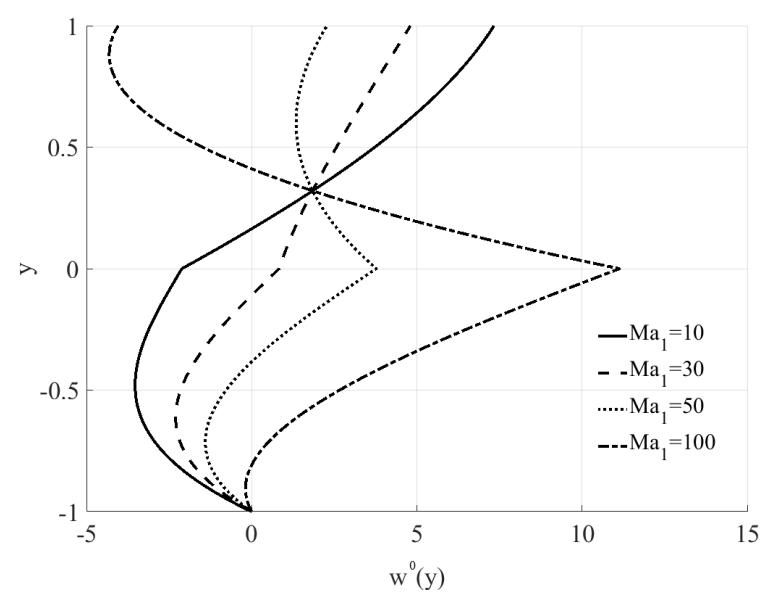

a

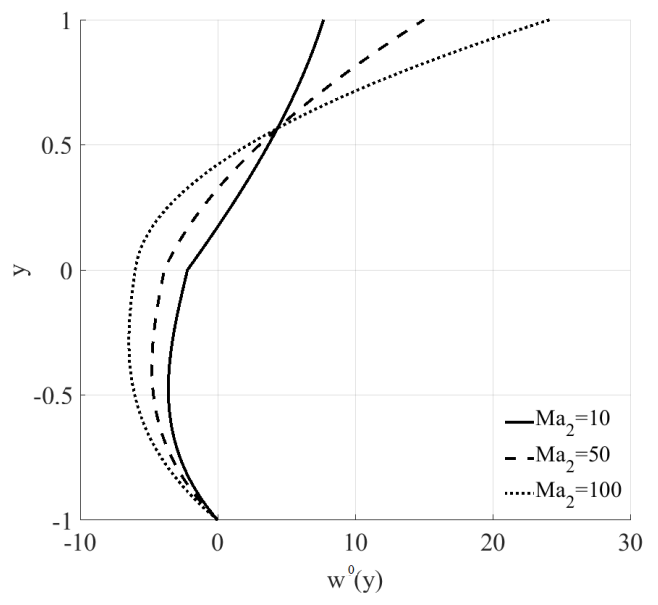

6

Fig. 4. Stationary profile of velocities $w_{j}^{0}(y)$ depending on the Marangoni numbers $\mathrm{Ma}_{1}$ (a) and $\mathrm{Ma}_{2}(\mathrm{~b})$

\section{Solution of conjugate problem by the Laplace transformation method. Analysis of numerical results}

To solve non-stationary problems (4)-(7) and (8)-(14) we apply the Laplace transform. As a result, we arrive to boundary value problems for images $A_{j}(y, s)$ of functions $a_{j}(y, t)$

$$
\begin{gathered}
A_{1 y y}(y, s)-s A_{1}(y, s)=-a_{10}(y), \quad-1<y<0, \\
A_{2 y y}(y, s)-s \chi h^{-2} A_{2}(y, s)=-\chi h^{-2} a_{20}(y), \quad 0<y<1, \\
A_{1}(-1, s)=A(s), \quad A_{2 y}(1, s)+\operatorname{Bi} A_{2}(1, s)=0, \\
A_{1}(0, s)=A_{2}(0, s), \quad k A_{1 y}(0, s)=h A_{2 y}(0, s)
\end{gathered}
$$


and images $W_{j}(y, s)$ of functions $w_{j}(y, t)$

$$
\begin{gathered}
W_{1 y y}(y, s)-s \mathrm{P}_{1}^{-1} W_{1}(y, s)=-\left(\mathrm{P}_{1}^{-1} w_{10}(y)+\operatorname{Ra}_{1} \int_{0}^{y} A_{1}(z, s) d z+\hat{C}(s)\right), \quad-1<y<0 \\
W_{2 y y}(y, s)-\frac{s \chi}{h^{2} \mathrm{P}_{2}} W_{2}(y, p)=-\left(\frac{\chi}{h^{2} \mathrm{P}_{2}} w_{20}(y)+\operatorname{Ra}_{2} \int_{0}^{y} A_{2}(z, s) d z+\frac{\mu \chi}{h^{3}} \hat{C}(s)\right), 0<y<1 \\
W_{1}(-1, s)=0, \quad \chi h^{-1} W_{1}(0, s)=W_{2}(0, s), \quad W_{2 y}(1, s)=\operatorname{Ma}_{1} A_{2}(1, s) \\
W_{2 y}(0, s)-\mu \chi h^{-2} W_{1 y}(0, s)=-\operatorname{Ma}_{1} A_{1}(0, s), \quad \chi \int_{-1}^{0} W_{2}(y, s) d y+\int_{0}^{1} W_{2}(y, s) d y=Q(s)
\end{gathered}
$$

When deriving equations (17), (19), the initial data (5), (10) were used. In (18), (20) the $A(s)$ and $Q(s)$ are images of the given functions $a(t)$ and $q(t)$ respectively (see conditions $(6),(14)$ ). The general solution of equations (17), (19) has the form

$$
\begin{gathered}
A_{1}(y, s)=b_{1} \operatorname{sh} \sqrt{s} y+b_{2} \operatorname{ch} \sqrt{s} y-\frac{1}{\sqrt{s}} \int_{0}^{y} a_{10}(z) \operatorname{sh} \sqrt{s}(y-z) d z \\
A_{2}(y, s)=b_{3} \operatorname{sh} \sqrt{s} y+b_{4} \operatorname{ch} \sqrt{s} y-\frac{\sqrt{\chi}}{h \sqrt{s}} \int_{0}^{y} a_{20}(z) \operatorname{sh} \sqrt{s}(y-z) d z \\
W_{1}(y, s)=d_{1} \operatorname{sh} \alpha_{1} y+d_{2} \operatorname{ch} \alpha_{1} y-\frac{1}{\alpha_{1}} \int_{0}^{y} f_{1}(z, s) \operatorname{sh} \alpha_{1}(y-z) d z-\frac{\hat{C}(s)}{\alpha_{1}}\left(\operatorname{ch} \alpha_{1} y-\frac{1}{\alpha_{1}}\right), \\
W_{2}(y, s)=d_{3} \operatorname{sh} \alpha_{2} y+d_{4} \operatorname{ch} \alpha_{2} y-\frac{1}{\alpha_{2}} \int_{0}^{y} f_{2}(z, p) \operatorname{sh} \alpha_{2}(y-z) d z-\frac{\mu \mathrm{P}_{2} \hat{C}(s)}{s h}\left(\operatorname{ch~} \alpha_{2} y-1\right), \\
f_{1}(y, p)=\mathrm{P}_{1}^{-1} w_{10}(y)+\operatorname{Ra}_{1} \int_{0}^{y} A_{1}(z, s) d z, \quad f_{2}(y, s)=\frac{\chi}{h^{2} \mathrm{P}_{2}} w_{20}(y)+\operatorname{Ra}_{2} \int_{0}^{y} A_{2}(z, s) d z \\
\alpha_{1}=\sqrt{s \mathrm{P}_{1}^{-1}}, \quad \alpha_{2}=\sqrt{s \chi \mathrm{P}_{2}^{-1}} h^{-1} .
\end{gathered}
$$

The values $m_{k}, d_{k}, k=\overline{1,4}$, appearing in (21), and function $\hat{C}(s)$ are determined from the boundary conditions (18), (20). The type of these values is not presented here because of its complexity.

Suppose, that $\lim _{t \rightarrow \infty} a(t)=a_{0}$ and $\lim _{t \rightarrow \infty} q(t)=q_{0}$. Using the obtained representations for $A_{j}(y, s), W_{j}(y, s)$ and $\hat{C}(p)$, we can prove the limit equalities

$$
\begin{gathered}
\lim _{t \rightarrow \infty} a_{j}(y, t)=\lim _{s \rightarrow 0} s A_{j}(y, s)=a_{j}^{s}(y), \lim _{t \rightarrow \infty} w_{j}(y, t)=\lim _{s \rightarrow 0} s W_{j}(y, s)=w_{j}^{s}(y), \\
\lim _{t \rightarrow \infty} C(t)=\lim _{s \rightarrow 0} s \hat{C}(s)=C^{s},
\end{gathered}
$$

where $a_{j}^{s}(y), w^{s}(y)$ and $C^{s}$ are given by formulas (15), (16).

Using the method of numerical inversion of the Laplace transform, we obtain some results for the velocities. The case when $q(t)=0$ (the flow rate is zero and the movement occurs only due to thermogravitational forces) is considered, and the longitudinal temperature gradient on the bottom wall is distributed according to the law $\left.a_{(} t\right)=a_{0}+\gamma_{1} e^{-\gamma_{2} t} \sin \left(\gamma_{3} t\right)$, where the coefficients $\gamma_{1}, \gamma_{2}$ are responsible for the amplitude and frequency of the oscillations, respectively. In the case when $a \neq 0, \gamma_{2}>0$, then, according to equalities (22), the solution converges to the stationary state (see Fig. $5 \mathrm{a}$ ), and for $\gamma_{2} \leqslant 0$, the limits of the functions $\left.a_{(} t\right)$ at $t \rightarrow \infty$ do not exist and the solution does not tend to the stationary state (see Fig. 5 b). As $a(t)$ discontinuous functions can also be specified, thereby also influencing the nature of the flow. 

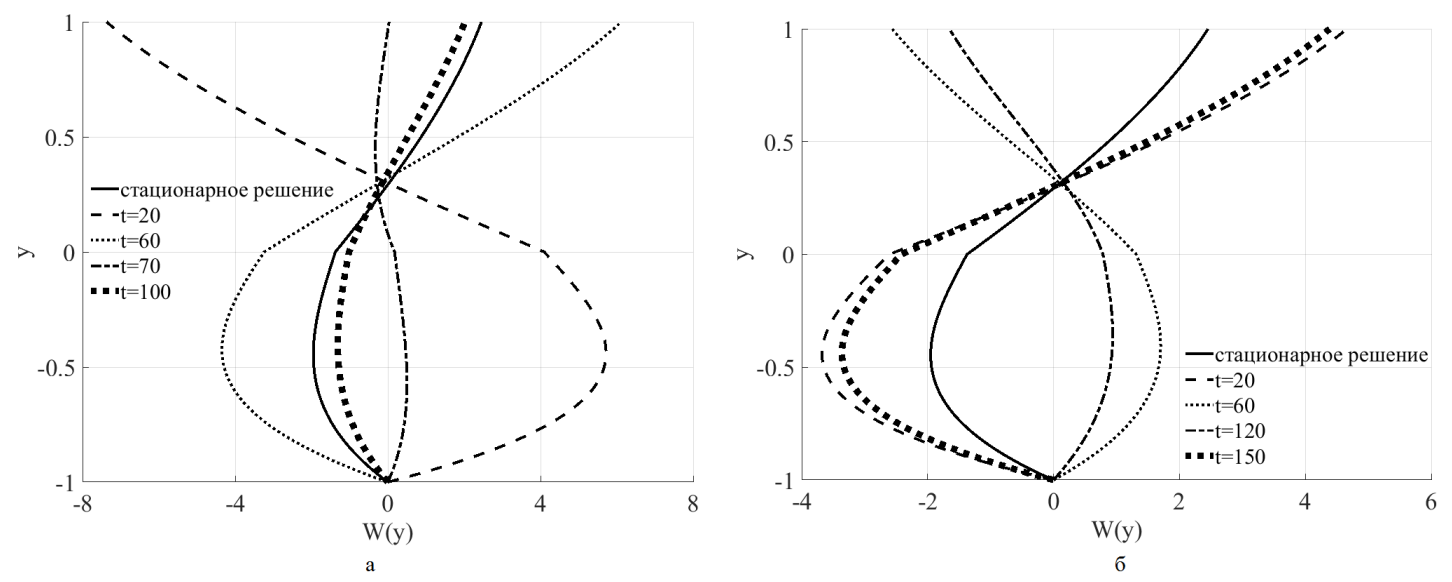

Fig. 5. Profile of dimensionless velocities $W_{j}(y)$ at $a(t)=1-5 e^{-0.01 t} \sin (0.1 t)$ (a) and $a(t)=2 \sin (0.1 t)(b)$

This work is supported by the Krasnoyarsk Mathematical Center and financed by the Ministry of Science and Higher Education of the Russian Federation in the framework of the establishment and development of regional Centers for Mathematics Research and Education (Agreement no. 075-02-2020-1631).

\section{References}

[1] M.K.Smith, S.H.Davis, Instabilities of dynamic thermocapillary liquid layers. Part 2. Surface wave instabillities, J. Fluid Mech., 132(1983), no. 7, 145-162.

[2] A.A.Vedenov, G.G.Gladush, Physical processes during laser processing of metals, Moscow, Nauka, 1985 (in Russian).

[3] E.A.Ryabitskii, Thermocapillarity instability of liquid layer with internal heat generation, Microgravity Science and Technology, 7(1994), no. 1, 20-23.

[4] H.J.Palmer, J.C.Berg, Hydrodynamic stability of surfactant solutions heated from below, $J$. Fluid Mech., 51(1972), no. 2, 385-402.

[5] R.Kh.Zeytovnian, Convection in Fluids, Dordrecht, Springer, 2009.

[6] R.V.Birikh, Thermocapillary convection in a horizontal layer of liquid, J. Appl. Mech. Tech. Phys., 5(1966), 69-72.

[7] V.K.Andreev, Yu.A.Gaponenko, O.N.Goncharova, V.V.Pukhnachev, Mathematical Models of Convection, Berlin/Boston, Walter de Gruyter GmbH and CO KG, 2012.

[8] V.V.Pukhnachov, Non-stationary Analogues of the Birikh Solution, Nauchnyi zhurnal teoreticheskih i prikladnyh issledovanii. Novosti Altaiskogo Gos. Universiteta, 69(2011), no. 1-2, 62-69 (in Russian).

[9] V.K.Andreev, Birikh solution of convection equations and some their generalizations, Preprint of ICM SB RAS, Krasnoyarsk, 2010 (in Russian). 
[10] V.K.Andreev, E.N.Lemeshkova, Linear problems of motions with interfaces, Krasnoyarsk, Sib. Feder. Univ., 2018 (in Russian).

[11] R.Kh.Zeytounian, The Benard-Marangoni thermocapillary-instability problem, Phys. Usp., 41(1998), 241-267.

\title{
Слоистое движение двух несмешивающихся жидкостей со свободной границей
}

Елена Н. Лемешкова

Институт вычислительного моделирования СО РАН

Красноярск, Российская Федерация

\begin{abstract}
Аннотация. Изучено однонаправленное движение двух вязких несжимаемых жидкостей в плоском канале. На нижней твердой стенке задан нестационарный градиент температуры, а верхняя стенка - свободная граница. Жидкости контактируют по плоской поверхности раздела. Движение вызвано совместным действием термогравитационных и термокапиллярных сил и заданного общего нестационарного расхода в слоях. Соответствующая начально-краевая задача является сопряжённой и обратной, поскольку градиент давления вдоль канала должен находиться вместе с полем скоростей и температур. Для нее найдено точное стационарное решение. В изображениях по Лапласу решение нестационарной задачи находится в виде квадратур. Установлено, что если температура на нижней стенке и расход стабилизируются со временем, то движение выходит на стационарный режим с ростом времени, что говорит об устойчивости стационарного решения относительно однонаправленных нестационарных возмущений. Приведены результаты расчетов, показывающие различные способы управления движением с помощью задания температуры на стенке.
\end{abstract}

Ключевые слова: термокапиллярность, поверхность раздела, уравнения Обербека-Буссинеска. 